\title{
A Range-Based Test for the Parametric Form of the Volatility in Diffusion Models.
}

\author{
Mark Podolskij* \\ Ruhr-Universität Bochum \\ Fakultät für Mathematik \\ 44780 Bochum, Germany \\ e-mail: podolski@cityweb.de
}

\author{
Daniel Ziggel \\ Ruhr-Universität Bochum \\ Fakultät für Mathematik \\ 44780 Bochum, Germany \\ e-mail: daniel.ziggel@rub.de
}

\begin{abstract}
We propose a new test for the parametric form of the volatility function in continuous time diffusion models of the type $d X_{t}=a\left(t, X_{t}\right) d t+\sigma\left(t, X_{t}\right) d W_{t}$. Our approach involves a range-based estimation of the integrated volatility and the integrated quarticity, which are used to construct the test statistic. Under rather weak assumptions on the drift and volatility we prove weak convergence of the test statistic to a centered mixed Gaussian distribution. As a consequence we obtain a test, which is consistent for any fixed alternative. Moreover, we present a parametric bootstrap procedure which provides a better approximation of the distribution of the test statistic. Finally, it is demonstrated by means of Monte Carlo study that the range-based test is more powerful than the return-based test when comparing at the same sampling frequency.
\end{abstract}

Keywords: Bipower Variation; Central Limit Theorem; Diffusion Models; Goodness-OfFit Testing; High-Frequency Data; Integrated Volatility; Range-Based Bipower Variation Semimartingale Theory 


\section{Introduction}

Continuous time stochastic processes are essential tools for theoretical asset and option pricing. Ito diffusions, which are solutions of the stochastic differential equation

$$
d X_{t}=a\left(t, X_{t}\right) d t+\sigma\left(t, X_{t}\right) d W_{t} \quad t \in[0,1]
$$

where the interval $[0,1]$ typically represents a trading day, constitute a commonly used class of processes for modelling the dynamics of asset prices or interest rates. Here $W$ denotes the Brownian motion, $a$ is the drift function and $\sigma$ is the volatility function, and the process $X=\left(X_{t}\right)_{t \in[0,1]}$ is assumed to be observed at time points $t_{i}=i / N, i=0, \ldots, N$.

In the last decades various parametric models have been proposed for different types of options (see, for instance, Black \& Scholes (1973), Vasicek (1977), Cox, Ingersoll \& Ross (1985), Karatzas (1988) or Constantinides (1992) among many others). An adequate modelling of the volatility function $\sigma$ in (1) is crucial for the option pricing and misspecification leads to serious errors in the data analysis.

Surprisingly, there are only few theoretical results on tests of the parametric form of the volatility in the high frequency setting (see Corradi \& White (1999), Dette \& von Lieres und Wilkau (2003), Dette, Podolskij \& Vetter (2006) and Dette \& Podolskij (2006)). In the following we present a consistent (range-based) test for the null hypothesis that the true volatility $\sigma^{2}$ lies in a vector space $\mathcal{U}$, which is spanned by given volatility functions

$$
\sigma_{1}^{2}, \ldots, \sigma_{d}^{2}:[0,1] \times \mathbb{R} \rightarrow \mathbb{R},
$$

based on the high frequency observations $X_{i / N}$. The key issue of our approach is the quantity

$$
M^{2}=\min _{\alpha_{1}, \ldots, \alpha_{d} \in \mathbb{R}^{d}} \int_{0}^{1}\left(\sigma^{2}\left(s, X_{s}\right)-\sum_{j=1}^{d} \alpha_{j} \sigma_{j}^{2}\left(s, X_{s}\right)\right)^{2} d s,
$$

which measures the $\mathcal{L}^{2}$-distance between $\sigma^{2}$ and $\mathcal{U}$. We construct a range-based estimator $M_{N}^{2}$ of $M^{2}$ and derive a central limit theorem for a standardised version of $M_{N}^{2}$. We reject the null hypothesis for large values of $M_{N}^{2}$.

This method is similar in spirit to the procedure proposed by Dette, Podolskij \& Vetter (2006), who estimated $M^{2}$ by means of return-based statistics (see also Dette \& Podolskij (2006) for an empirical process approach). However, practitioners usually use return-based statistics based on moderate frequencies, say 5- or 10-minutes frequency, due to microstructure noise which contaminates the true price process (indeed, Hansen \& Lunde (2006) demonstrate empirically that the microstructure noise can be ignored at moderate frequencies). Instead of using 5- or 10-minutes returns we propose to use 5- or 10-minutes ranges which turn out to be much more efficient. For this reason we expect to obtain a more powerful test than the one proposed by Dette, Podolskij \& Vetter (2006). 
This paper is organised as follows. In Section 2 we state the main assumptions and define the range-based estimator $M_{N}^{2}$ of the distance measure $M^{2}$. Furthermore, we prove a stable central limit theorem for a standardised version of $M_{N}^{2}$. As a result we obtain a consistent test of the hypothesis that $\sigma^{2} \in \mathcal{U}$. In Section 3 we study the finite sample performance of our test by means of Monte Carlo simulations. Moreover, we introduce a parametric bootstrap procedure which improves the power of the test. Finally, we demonstrate the proofs of the asymptotic results in the Appendix.

\section{Set-up and test procedure}

We start with a filtered probability space $\left(\Omega, \mathcal{F},\left(\mathcal{F}_{t}\right)_{t \in[0,1]}, P\right)$. Here and throughout this paper we assume that the stochastic differential equation (1) admits a unique strong solution $X=$ $\left(X_{t}\right)_{t \in[0,1]}$ with starting value $X_{0}$ ( $X_{0}$ is assumed to be deterministic), which is adapted to the filtration $\left(\mathcal{F}_{t}\right)_{t \in[0,1]}$ (see e.g. Karatzas \& Shreve (1991) p. 289 for sufficient conditions for existence and uniqueness of a strong solution). In this case the process $X$ has a representation

$$
X_{t}=X_{0}+\int_{0}^{t} a\left(s, X_{s}\right) d s+\int_{0}^{t} \sigma\left(s, X_{s}\right) d W_{s}, \quad t \in[0,1] .
$$

In this article we construct a new test for the hypothesis that the true volatility function $\sigma^{2}$ can be represented as a linear combination of the functions $\sigma_{1}^{2}, \ldots, \sigma_{d}^{2}$ given by (2), i.e.

$$
\sigma^{2}\left(t, X_{t}\right)=\sum_{j=1}^{d} \alpha_{j} \sigma_{j}^{2}\left(t, X_{t}\right) \quad \forall t \in[0,1],
$$

for some $\alpha=\left(\alpha_{1}, \ldots, \alpha_{d}\right)^{T} \in \mathbb{R}^{d}$, along the observed path $\left(X_{t}(\omega)\right)_{t \in[0,1]}$.

Remark 1 Let us consider an ideal situation of a complete observation of the path $\left(X_{t}\right)_{t \in[0,1]}$. In this case we can of course tell whether (5) is true or not, but it is impossible to decide whether the function $\sigma^{2}$ can be represented as a linear combination of the functions $\sigma_{1}^{2}, \ldots, \sigma_{d}^{2}$ outside the set $\left\{X_{t}(\omega) \mid t \in[0,1]\right\}$ or not. However, when the fuctions $\sigma^{2}, \sigma_{1}^{2}, \ldots, \sigma_{d}^{2}$ are analytic on $[0,1] \times \mathbb{R}$, then verifying (5) is obviously enough to conclude the corresponding assertion for all $(t, x) \in[0,1] \times \mathbb{R}$ (if the observed path of $X$ is not constant).

Note that the representation (5) holds if and only if $M^{2}=0$ along the observed path $\left(X_{t}(\omega)\right)_{t \in[0,1]}$. Consequently, we define the null hypothesis and the alternative by

$$
H_{0}: \omega \in \Omega_{0}=\left\{\omega \in \Omega \mid M^{2}(\omega)=0\right\}, \quad H_{1}: \omega \in \Omega_{1}=\left\{\omega \in \Omega \mid M^{2}(\omega)>0\right\},
$$

respectively.

Before we proceed with the discussion of the testing procedure we state some assumptions which are required to prove the asymptotic results of this paper. 
(A1) The processes $a_{t}=a\left(t, X_{t}\right), \sigma_{t}=\sigma\left(t, X_{t}\right), \sigma_{1 t}=\sigma_{1}\left(t, X_{t}\right), \ldots, \sigma_{d t}=\sigma_{d}\left(t, X_{t}\right)$ are continuous.

(A2) The assumption (A1) holds and $\sigma \in C^{1,2}([0,1] \times \mathbb{R})$ (i.e. the function $\sigma$ is once continuously differentiable in $t$ and twice continuously differentiable in $x$ ). Furthermore, the process $\sigma_{t}$ does not vanish.

(A3) The functions $\sigma_{1}^{2}, \ldots, \sigma_{d}^{2}$ are linearly independent on $[0,1] \times[a, b]$ for any $a<b$.

Next, we demonstrate an equivalent representation of the quantity $M^{2}$ given in (3). For this purpose we define an inner product for (square integrable) functions $f, g:[0,1] \times \mathbb{R} \rightarrow \mathbb{R}$ by

$$
[f, g]=\int_{0}^{1} f\left(s, X_{s}\right) g\left(s, X_{s}\right) d s .
$$

Notice that $M^{2}$ is the $\mathcal{L}^{2}$-distance (induced by $[\cdot, \cdot]$ ) between the function $\sigma^{2}$ and the vector space spanned by $\sigma_{1}^{2}, \ldots, \sigma_{d}^{2}$. By standard arguments of the Hilbert space theory (see e.g. Achieser (1956)) we immediately obtain the identity

$$
M^{2}=C_{0}-\left(C_{1}, \ldots, C_{d}\right) D^{-1}\left(C_{1}, \ldots, C_{d}\right)^{T},
$$

where the quantities $C_{0}, C_{1}, \ldots, C_{d}$ and $D=\left(D_{i j}\right)_{1 \leq i, j \leq d}$ are given by

$$
\begin{aligned}
& C_{0}=\left[\sigma^{2}, \sigma^{2}\right]=\int_{0}^{1} \sigma^{4}\left(s, X_{s}\right) d s, \\
& C_{i}=\left[\sigma_{i}^{2}, \sigma^{2}\right]=\int_{0}^{1} \sigma_{i}^{2}\left(s, X_{s}\right) \sigma^{2}\left(s, X_{s}\right) d s, \quad 1 \leq i \leq d, \\
& D_{i j}=\left[\sigma_{i}^{2}, \sigma_{j}^{2}\right]=\int_{0}^{1} \sigma_{i}^{2}\left(s, X_{s}\right) \sigma_{j}^{2}\left(s, X_{s}\right) d s, \quad 1 \leq i, j \leq d .
\end{aligned}
$$

Note that assumption (A3) implies the invertibility of the random matrix $D$ as long as the process $X$ is not constant with positive probability.

In the following we use the range-based method discussed in Christensen \& Podolskij (2006a,b) to construct the empirical analogues of the terms defined in (7). Let us briefly recall the basic ideas of this concept.

\subsection{Range-based estimation}

Assume that $N=n m$ with $n$ and $m$ being two natural numbers. The observed range of the process $X$ on the interval $\left[\frac{i-1}{n}, \frac{i}{n}\right]$ is defined by

$$
s_{i, n, m}=\sup _{s, t \in\left[\frac{i-1}{n}, \frac{i}{n}\right] \cap I_{N}}\left|X_{t}-X_{s}\right|
$$


with $I_{N}=\{j / N \mid j=0, \ldots, N\}$. We write $s_{i, n, m}(X)$ if we want to emphasise the dependence on the process $X$. Note that the range statistic $s_{i, n, m}$ is based on $m+1$ observations recorded in the interval $\left[\frac{i-1}{n}, \frac{i}{n}\right]$, and when $m=1$ we simply obtain the absolute returns.

The core of the range-based approach is the class of the realised range-based power variation given by $(r \geq 0)$

$$
R P V(r)_{n, m}=n^{\frac{r}{2}-1} \sum_{i=1}^{n} s_{i, n, m}^{r}
$$

Theorem 1 Assume that (A1) holds. As $n \rightarrow \infty$ we obtain

$$
\left.\sup _{m}\left|R P V(r)_{n, m}-\lambda_{r, m} \int_{0}^{1}\right| \sigma\left(s, X_{s}\right)\right|^{r} d s \mid \stackrel{P}{\longrightarrow} 0
$$

where the constant $\lambda_{r, m}$ is defined by

$$
\lambda_{r, m}=E\left[\sup _{s, t \in[0,1] \cap I_{m}}\left|W_{t}-W_{s}\right|^{r}\right] .
$$

Proof: See Christensen \& Podolskij (2006b).

Remark 2 To the best of our knowledge there is no explicit expression for $\lambda_{r, m}$ (however, it can be easily computed by simulations). When " $m=\infty$ " the constant $\lambda_{r}=\lim _{m \rightarrow \infty} \lambda_{r, m}$ is obviously the rth moment of the range of the Brownian motion on the interval [0,1]. Feller (1951) showed that the density function of $R=\sup _{s, t \in[0,1]}\left|W_{t}-W_{s}\right|$ can be represented as

$$
\delta(x)=8 \sum_{k=1}^{\infty}(-1)^{k+1} k^{2} \phi(k x),
$$

where $\phi$ denotes the density function of a standard normal distribution. Using this formula we can deduce that $\lambda_{r}<\infty$ for all $r \in \mathbb{R}$ (!) and for $r \geq 1$ we have

$$
\begin{aligned}
& \lambda_{r}=\frac{2^{\frac{r}{2}+1}(r-1)}{\sqrt{\pi}} \Gamma\left(\frac{r-1}{2}\right) \sum_{k=1}^{\infty} \frac{(-1)^{k+1}}{k^{r-1}} \quad r>1, \\
& \lambda_{1}=\frac{2 \sqrt{2}}{\sqrt{\pi}} .
\end{aligned}
$$

We refer to Podolskij (2006) for a detailed proof.

Theorem 1 already gives a hint how to construct the estimates of the terms defined in (7). However, we need the asymptotic distribution theory to derive a test for the parametric form of the volatility.

In the following discussion we will intensively use the concept of stable convergence. Let us shortly recall the definition. A sequence of random variables $Y_{n}$ converges stably in law with limit $Y$ (throughout this paper we write $Y_{n} \stackrel{\mathcal{D}_{s t}}{\longrightarrow} Y$ ), defined on an appropriate extension 
$\left(\Omega^{\prime}, \mathcal{F}^{\prime}, P^{\prime}\right)$ of the probability space $(\Omega, \mathcal{F}, P)$, if and only if for any $\mathcal{F}$-measurable and bounded random variable $Z$ and any bounded and continuous function $g$ the convergence

$$
\lim _{n \rightarrow \infty} E\left[Z g\left(Y_{n}\right)\right]=E[Z g(Y)]
$$

holds. This is obviously a slightly stronger mode of convergence than convergence in law (see Renyi (1963) or Aldous \& Eagleson (1978) for more details on stable convergence).

Theorem 2 Assume that (A2) holds. As $n \rightarrow \infty$ (and $m$ is fixed) we have

$$
\sqrt{n}\left(R P V(r)_{n, m}-\lambda_{r, m} \int_{0}^{1}\left|\sigma\left(s, X_{s}\right)\right|^{r} d s\right) \stackrel{\mathcal{D}_{s t}}{\longrightarrow} \sqrt{\lambda_{2 r, m}-\lambda_{r, m}^{2}} \int_{0}^{1}\left|\sigma\left(s, X_{s}\right)\right|^{r} d W_{s}^{\prime},
$$

where $W^{\prime}$ denotes another Brownian motion defined on an extension of the filtered probability space $\left(\Omega, \mathcal{F},\left(\mathcal{F}_{t}\right)_{t \in[0,1]}, P\right)$ and independent of the $\sigma$-field $\mathcal{F}$.

Proof: See Christensen \& Podolskij (2006b).

Even though we have formulated Theorem 2 for a fixed $m$ it also holds for the case " $m=\infty$ ".

Notice that assumption (A2) and the Ito's formula imply that the volatility process $\left(\sigma_{t}\right)$ is an Ito diffusion, which is in turn a standard condition for stable central limit theorems in high frequency setting (see, for instance, Barndorff-Nielsen, Graversen, Jacod, Podolskij \& Shephard (2006) or Christensen \& Podolskij (2006a,b)).

From Theorem 1 we can conclude that the statistic $\lambda_{2, m}^{-1} R P V(2)_{n, m}$ is a consistent estimator of the quantity $\int_{0}^{1} \sigma^{2}\left(s, X_{s}\right) d s$, which is often called the integrated volatility in the econometric literature, whereas Theorem 2 gives the central limit theorem for this statistic. Note also that $\int_{0}^{1} \sigma^{2}\left(s, X_{s}\right) d s$ is the quadratic variation of the process $X$ at time 1 . Now let us compare the efficiency of the above range-based estimator with the efficiency of the "usual" estimator of the integrated volatility. Recall that the estimator

$$
R V_{n}=\sum_{i=1}^{n}\left(X_{\frac{i}{n}}-X_{\frac{i-1}{n}}\right)^{2},
$$

which is often referred to as the realised volatility, is also a consistent estimator of the integrated volatility. In fact, it is a very natural estimator because $R V_{n}$ converges in probability to $\int_{0}^{1} \sigma^{2}\left(s, X_{s}\right) d s$ by the definition of the quadratic variation. From Barndorff-Nielsen, Graversen, Jacod, Podolskij \& Shephard (2006) and Theorem 2 we deduce the following stable central limit theorems:

$$
\begin{aligned}
& \sqrt{n}\left(R V_{n}-\int_{0}^{1} \sigma^{2}\left(s, X_{s}\right) d s\right) \stackrel{\mathcal{D}_{s t}}{\longrightarrow} M N\left(0,2 \int_{0}^{1} \sigma^{4}\left(s, X_{s}\right) d s\right), \\
& \sqrt{n}\left(\lambda_{2, m}^{-1} R P V(2)_{n, m}-\int_{0}^{1} \sigma^{2}\left(s, X_{s}\right) d s\right) \stackrel{\mathcal{D}_{s t}}{\longrightarrow} M N\left(0, \frac{\lambda_{4, m}-\lambda_{2, m}^{2}}{\lambda_{2, m}^{2}} \int_{0}^{1} \sigma^{4}\left(s, X_{s}\right) d s\right) .
\end{aligned}
$$


Here $M N\left(0, \eta^{2}\right)$ denotes the mixed normal distribution with conditional variance $\eta^{2}$ (in fact, the above stable convergence is an equivalent way of determining the stable limit). In Christensen $\&$ Podolskij (2006a) we have shown that

$$
\lim _{m \rightarrow \infty} \frac{\lambda_{4, m}-\lambda_{2, m}^{2}}{\lambda_{2, m}^{2}} \approx 0.4, \quad \frac{\lambda_{4, m}-\lambda_{2, m}^{2}}{\lambda_{2, m}^{2}} \leq 2 \quad \text { for all } m .
$$

This means that the range-based estimator $\lambda_{2, m}^{-1} R P V(2)_{n, m}$ is more efficient than $R V_{n}$.

Note, however, that statistically the above comparison is unfair, because $\lambda_{2, m}^{-1} R P V(2)_{n, m}$ is based on $n m$ observations whereas $R V_{n}$ is based only on $n$ observations (actually $R V_{n}$ is known to be the maximum likelihood estimator of the integrated volatility when the drift function $a$ is zero and the volatility is constant). Nevertheless, the range-based estimator $\lambda_{2, m}^{-1} R P V(2)_{n, m}$ has some practical advantages. It is widely accepted in the econometric literature that the price process $X$ is contaminated by microstructure noise (see e.g. Barndorff-Nielsen \& Shephard (2007)). This means that the realised volatility becomes an inadequate measure of the integrated volatility when using all observed data. For this reason practitioners usually use moderate frequencies, like 5 - or 10-minutes frequencies, to compute the realised volatility, since the influence of the noise at those frequencies can be ignored (see Hansen \& Lunde (2006) for empirical justification of this procedure). Christensen \& Podolskij (2006a) have proposed to use 5- or 10-minutes ranges instead of 5- or 10-minutes returns to construct an estimator of the integrated volatility (see also Martens \& van Dijk (2005) for empirical comparison between the range-based and return-based approach). The above-mentioned central limit theorems approve the advantages of the range-based method.

\subsection{Testing procedure}

We are now in a position to construct consistent estimates of the quantities $C_{0}, C_{1}, \ldots, C_{d}$ and $D$ defined by (7). We consider the statistics

$$
\begin{aligned}
& C_{0}^{n, m}=\lambda_{4, m}^{-1} n \sum_{k=1}^{n} s_{k, n, m}^{4} \\
& C_{i}^{n, m}=\lambda_{2, m}^{-1} \sum_{k=1}^{n} \sigma_{i}^{2}\left(\frac{k-1}{n}, X_{\frac{k-1}{n}}\right) s_{k, n, m}^{2}, \quad 1 \leq i \leq d, \\
& D_{i j}^{n}=\frac{1}{n} \sum_{k=1}^{n} \sigma_{i}^{2}\left(\frac{k-1}{n}, X_{\frac{k-1}{n}}\right) \sigma_{j}^{2}\left(\frac{k-1}{n}, X_{\frac{k-1}{n}}\right), \quad 1 \leq i, j \leq d .
\end{aligned}
$$

Under assumption (A1) $C_{0}^{n, m}, C_{1}^{n, m}, \ldots, C_{d}^{n, m}$ and $D^{n}$ converge in probability to $C_{0}, C_{1}, \ldots, C_{d}$ and $D$, respectively, and we obtain

$$
M_{N}^{2}=C_{0}^{n, m}-\left(C_{1}^{n, m}, \ldots, C_{d}^{n, m}\right)\left(D^{n}\right)^{-1}\left(C_{1}^{n, m}, \ldots, C_{d}^{n, m}\right)^{T}
$$


as a consistent estimator of the distance measure $M^{2}$. The next theorem demonstrates the stable convergence of the vector $\sqrt{n}\left(C_{0}^{n, m}-C_{0}, C_{1}^{n, m}-C_{1}, \ldots, C_{d}^{n, m}-C_{d}\right)^{T}$.

Theorem 3 Assume that conditions (A2) and (A3) holds. As $n \rightarrow \infty$ (and $m$ is fixed) we have

$$
D^{n}-D=o_{p}\left(n^{-\frac{1}{2}}\right)
$$

and

$$
\sqrt{n}\left(\begin{array}{c}
C_{0}^{n, m}-C_{0} \\
C_{1}^{n, m}-C_{1} \\
\vdots \\
C_{d}^{n, m}-C_{d}
\end{array}\right) \stackrel{\mathcal{D}_{s t}}{\longrightarrow} \int_{0}^{1} \Sigma_{s}^{\frac{1}{2}} d W_{s}^{\prime}
$$

where $\Sigma_{s}$ is $(d+1)$-dimensional matrix given by

$$
\Sigma_{s}=\left(\begin{array}{cccc}
v(s) & v_{1}(s) & \cdots & v_{d}(s) \\
v_{1}(s) & v_{11}(s) & \cdots & v_{1 d}(s) \\
\vdots & \vdots & \ddots & \vdots \\
v_{d}(s) & v_{d 1}(s) & \cdots & v_{d d}(s)
\end{array}\right)
$$

with

$$
\begin{aligned}
& v(s)=\frac{\lambda_{8, m}-\lambda_{4, m}^{2}}{\lambda_{4, m}^{2}} \sigma^{8}\left(s, X_{s}\right), \\
& v_{i}(s)=\frac{\lambda_{6, m}-\lambda_{4, m} \lambda_{2, m}}{\lambda_{4, m} \lambda_{2, m}} \sigma_{i}^{2}\left(s, X_{s}\right) \sigma^{6}\left(s, X_{s}\right), \quad 1 \leq i \leq d \\
& v_{i j}(s)=\frac{\lambda_{4, m}-\lambda_{2, m}^{2}}{\lambda_{2, m}^{2}} \sigma_{i}^{2}\left(s, X_{s}\right) \sigma_{j}^{2}\left(s, X_{s}\right) \sigma^{4}\left(s, X_{s}\right), \quad 1 \leq i, j \leq d,
\end{aligned}
$$

and $W^{\prime}$ is a $(d+1)$-dimensional Brownian motion defined on an extension of the filtered probability space $\left(\Omega, \mathcal{F},\left(\mathcal{F}_{t}\right)_{t \in[0,1]}, P\right)$ and independent of the $\sigma$-field $\mathcal{F}$.

Proof: see Appendix.

As we have already mentioned we can alternatively identify the stable limit in Theorem 3 as being mixed normal with conditional variance $\int_{0}^{1} \Sigma_{s} d s$. By a $\Delta$-method for stable convergence (see Podolskij (2006) for more details on the $\Delta$-method) we deduce the following result.

Corollary 1 Assume that conditions (A2) and (A3) hold. As $n \rightarrow \infty$ (and $m$ is fixed) we have

$$
\sqrt{n}\left(M_{N}^{2}-M^{2}\right) \stackrel{\mathcal{D}_{s t}}{\longrightarrow}\left(1,-2\left(C_{1}, \ldots, C_{d}\right) D^{-1}\right) \int_{0}^{1} \Sigma_{s}^{\frac{1}{2}} d W_{s}^{\prime},
$$

where $W^{\prime}$ is a $(d+1)$-dimensional Brownian motion defined on an extension of the filtered probability space $\left(\Omega, \mathcal{F},\left(\mathcal{F}_{t}\right)_{t \in[0,1]}, P\right)$ and independent of the $\sigma$-field $\mathcal{F}$. 
Now we demonstrate how Corollary 1 can be applied in order to obtain a feasible (standard) central limit theorem. Notice that the stable limit in (21) is mixed normal with conditional variance

$$
\rho^{2}=\left(1,-2\left(C_{1}, \ldots, C_{d}\right) D^{-1}\right) \int_{0}^{1} \Sigma_{s} d s\left(1,-2\left(C_{1}, \ldots, C_{d}\right) D^{-1}\right)^{T}
$$

Using again a version of Theorem 1 we obtain

$$
\rho_{N}^{2}=\left(1,-2\left(C_{1}^{n, m}, \ldots, C_{d}^{n, m}\right)\left(D^{n}\right)^{-1}\right) \Sigma_{N}\left(1,-2\left(C_{1}^{n, m}, \ldots, C_{d}^{n, m}\right)\left(D^{n}\right)^{-1}\right)^{T},
$$

where $\Sigma_{N}$ is given by

$$
\Sigma_{N}=\left(\begin{array}{cccc}
v^{N} & v_{1}^{N} & \cdots & v_{d}^{N} \\
v_{1}^{N} & v_{11}^{N} & \cdots & v_{1 d}^{N} \\
\vdots & \vdots & \ddots & \vdots \\
v_{d}^{N} & v_{d 1}^{N} & \cdots & v_{d d}^{N}
\end{array}\right)
$$

with

$$
\begin{aligned}
& v^{N}=\frac{\lambda_{8, m}-\lambda_{4, m}^{2}}{\lambda_{4, m}^{2} \lambda_{8, m}} n^{3} \sum_{k=1}^{n} s_{k, n, m}^{8}, \\
& v_{i}^{N}=\frac{\lambda_{6, m}-\lambda_{4, m} \lambda_{2, m}}{\lambda_{4, m} \lambda_{2, m} \lambda_{6, m}} n^{2} \sum_{k=1}^{n} \sigma_{i}^{2}\left(\frac{k-1}{n}, X_{\frac{k-1}{n}}\right) s_{k, n, m}^{6}, \quad 1 \leq i \leq d \\
& v_{i j}^{N}=\frac{\lambda_{4, m}-\lambda_{2, m}^{2}}{\lambda_{2, m}^{2} \lambda_{4, m}} n \sum_{k=1}^{n} \sigma_{i}^{2}\left(\frac{k-1}{n}, X_{\frac{k-1}{n}}\right) \sigma_{j}^{2}\left(\frac{k-1}{n}, X_{\frac{k-1}{n}}\right) s_{k, n, m}^{4}, \quad 1 \leq i, j \leq d,
\end{aligned}
$$

as a consistent estimator of $\rho^{2}$. The properties of stable convergence immediately imply the stable convergence

$$
\frac{\sqrt{n}\left(M_{N}^{2}-M^{2}\right)}{\rho_{N}} \stackrel{\mathcal{D}_{s t}}{\longrightarrow} W_{1}^{\prime} .
$$

With this result in hand we are able to test for the parametric form of the volatility function. The null hypothesis $H_{0}: \omega \in \Omega_{0}$ is rejected at level $\alpha$ when

$$
\frac{\sqrt{n} M_{N}^{2}}{\rho_{N}}>c_{1-\alpha}
$$

where $c_{1-\alpha}$ denotes the $(1-\alpha)$-quantile of a standard normal distribution. Indeed, the independence between $W^{\prime}$ and $\mathcal{F}$ implies the convergence

$$
P\left(\frac{\sqrt{n} M_{N}^{2}}{\rho_{N}}>c_{1-\alpha} \mid \Omega_{0}\right) \rightarrow \alpha .
$$

On the other hand it holds that

$$
P\left(\frac{\sqrt{n} M_{N}^{2}}{\rho_{N}}>c_{1-\alpha} \mid \Omega_{1}\right) \rightarrow 1,
$$

and consequently the resulting test is consistent against any fixed alternative. 
Remark 3 In practice, it is quite unrealistic to expect the true volatility function $\sigma^{2}$ to be representable as a linear combination of the given functions $\sigma_{1}^{2}, \ldots, \sigma_{d}^{2}$. A much more interesting question is: how close is $\sigma^{2}$ to the vector space spanned by $\sigma_{1}^{2}, \ldots, \sigma_{d}^{2}$ ? Notice, however, that the quantity $M^{2}$ is not an adequate measure for this problem until we put it in relation to the norm of $\sigma^{2}$ induced by $[\cdot, \cdot]$. This leads to precise neighborhood hypotheses

$H_{0}: \omega \in \Omega_{0}(\epsilon)=\left\{\omega \in \Omega \mid \frac{M^{2}(\omega)}{\left[\sigma^{2}, \sigma^{2}\right](\omega)} \leq \epsilon\right\}, \quad H_{1}: \omega \in \Omega_{1}(\epsilon)=\left\{\omega \in \Omega \mid \frac{M^{2}(\omega)}{\left[\sigma^{2}, \sigma^{2}\right](\omega)}>\epsilon\right\}$

where $0<\epsilon<1$ is a given constant (recall that $\left[\sigma^{2}, \sigma^{2}\right]=C_{0}=\int_{0}^{1} \sigma_{s}^{4} d s$ ). Hypotheses of this type have been discussed by Hodges $\&$ Lehmann (1954) in a classical setting.

By Theorem 3 and the $\Delta$-method for stable convergence we obtain

$$
\sqrt{n}\left(\frac{M_{N}^{2}}{C_{0}^{n, m}}-\frac{M^{2}}{C_{0}}\right) \stackrel{\mathcal{D}_{s t}}{\longrightarrow} M N\left(0, \tilde{\rho}^{2}\right),
$$

with

$$
\begin{aligned}
\tilde{\rho}^{2} & =\left(\frac{\left(C_{1}, \ldots, C_{d}\right) D^{-1}\left(C_{1}, \ldots, C_{d}\right)^{T}}{C_{0}^{2}}, \frac{-2\left(C_{1}, \ldots, C_{d}\right) D^{-1}}{C_{0}}\right) \int_{0}^{1} \Sigma_{s} d s \\
& \times\left(\frac{\left(C_{1}, \ldots, C_{d}\right) D^{-1}\left(C_{1}, \ldots, C_{d}\right)^{T}}{C_{0}^{2}}, \frac{-2\left(C_{1}, \ldots, C_{d}\right) D^{-1}}{C_{0}}\right)^{T} .
\end{aligned}
$$

The conditional variance $\tilde{\rho}^{2}$ can be consistently estimated by

$$
\begin{aligned}
\tilde{\rho}_{N}^{2} & =\left(\frac{\left(C_{1}^{n, m}, \ldots, C_{d}^{n, m}\right)\left(D^{n}\right)^{-1}\left(C_{1}^{n, m}, \ldots, C_{d}^{n, m}\right)^{T}}{\left(C_{0}^{n, m}\right)^{2}}, \frac{-2\left(C_{1}^{n, m}, \ldots, C_{d}^{n, m}\right)\left(D^{n}\right)^{-1}}{C_{0}^{n, m}}\right) \Sigma_{N} \\
& \times\left(\frac{\left(C_{1}^{n, m}, \ldots, C_{d}^{n, m}\right)\left(D^{n}\right)^{-1}\left(C_{1}^{n, m}, \ldots, C_{d}^{n, m}\right)^{T}}{\left(C_{0}^{n, m}\right)^{2}}, \frac{-2\left(C_{1}^{n, m}, \ldots, C_{d}^{n, m}\right)\left(D^{n}\right)^{-1}}{C_{0}^{n, m}}\right)^{T}
\end{aligned}
$$

Consequently, the null hypothesis $H_{0}: \omega \in \Omega_{0}(\epsilon)$ is rejected at level $\alpha$ when

$$
\frac{\sqrt{n}\left(\frac{M_{N}^{2}}{C_{0}^{n, m}}-\epsilon\right)}{\tilde{\rho}_{N}}>c_{1-\alpha}
$$

Note that the independence between $W^{\prime}$ and $\mathcal{F}$ implies

$$
P\left(\frac{\sqrt{n}\left(\frac{M_{N}^{2}}{C_{0}^{n, m}}-\epsilon\right)}{\tilde{\rho}_{N}}>c_{1-\alpha} \mid \Omega_{0}(\epsilon)\right) \leq P\left(\frac{\sqrt{n}\left(\frac{M_{N}^{2}}{C_{0}^{n, m}}-\frac{M^{2}}{C_{0}}\right)}{\tilde{\rho}_{N}}>c_{1-\alpha} \mid \Omega_{0}(\epsilon)\right) \rightarrow \alpha
$$

and

$$
P\left(\frac{\sqrt{n}\left(\frac{M_{N}^{2}}{C_{0}^{n, m}}-\epsilon\right)}{\tilde{\rho}_{N}}>c_{1-\alpha} \mid \Omega_{1}(\epsilon)\right) \rightarrow 1 .
$$

As a consequence of the latter convergence the test is consistent against any alternative. 


\section{Parametric bootstrap and finite sample properties}

\subsection{Parametric bootstrap}

In order to improve the finite sample properties we introduce a parametric bootstrap procedure. Throughout this subsection we assume that the functions $\sigma^{2}, \sigma_{1}^{2}, \ldots, \sigma_{d}^{2}$ are analytic on $[0,1] \times$ $\mathbb{R}$. In a first step we consider a coefficient vector $\alpha^{\text {min }}=\left(\alpha_{1}^{\min }, \ldots, \alpha_{d}^{\min }\right)^{T}$ defined by

$$
\alpha^{\min }=\operatorname{argmin}_{\left(\alpha_{1}, \ldots, \alpha_{d}\right)^{T} \in \mathbb{R}^{d}} \int_{0}^{1}\left(\sigma^{2}\left(s, X_{s}\right)-\sum_{j=1}^{d} \alpha_{j} \sigma_{j}^{2}\left(s, X_{s}\right)\right)^{2} d s .
$$

Applying Hilbert space arguments we obtain the representation

$$
\alpha^{\min }=D^{-1}\left(C_{1}, \ldots, C_{d}\right)^{T}
$$

where the quantities $D$ and $C$ are defined by (7), and consequently a consistent estimator of $\alpha^{\text {min }}$ is given by

$$
\alpha_{N}^{\min }=\left(D^{n}\right)^{-1}\left(C_{1}^{n, m}, \ldots, C_{d}^{n, m}\right)^{T} .
$$

In a second step we generate the data $X_{\frac{i}{N}}^{* j}(i=0, \ldots, N, j=1, \ldots, B)$ from the stochastic differential equation

$$
d X_{t}=\sum_{j=1}^{d} \alpha_{j N}^{m i n} \sigma_{j}\left(t, X_{t}\right) d W_{t}, \quad t \in[0,1]
$$

(recall that the drift function $a$ does not influence the distribution theory) and compute the bootstrap analogues

$$
\frac{\sqrt{n} M_{N}^{2}(1)}{\rho_{N}(1)}, \ldots, \frac{\sqrt{n} M_{N}^{2}(B)}{\rho_{N}(B)}
$$

of the statistic $\sqrt{n} M_{N}^{2} / \rho_{N}$. Finally, the value of $\sqrt{n} M_{N}^{2} / \rho_{N}$ is compared with the corresponding quantiles of the bootstrap distribution. Note that this bootstrap procedure makes sense due to the fact that the functions $\sigma^{2}, \sigma_{1}^{2}, \ldots, \sigma_{d}^{2}$ are analytic on $[0,1] \times \mathbb{R}$.

In the following we compare the finite sample performance of the range-based test with one proposed by Dette, Podolskij \& Vetter (2006). Let us briefly recall the construction of the test proposed in this article. The distance measure $M^{2}$ is estimated by the return-based statistic

$$
\tilde{M}_{n}^{2}=\tilde{C}_{0}^{n}-\left(\tilde{C}_{1}^{n}, \ldots, \tilde{C}_{d}^{n}\right)\left(D^{n}\right)^{-1}\left(\tilde{C}_{1}^{n}, \ldots, \tilde{C}_{d}^{n}\right)^{T}
$$

with

$$
\begin{aligned}
& \tilde{C}_{0}^{n}=\frac{n}{3} \sum_{k=1}^{n}\left(X_{\frac{k}{n}}-X_{\frac{k-1}{n}}\right)^{4}, \\
& \tilde{C}_{i}^{n}=\sum_{k=1}^{n} \sigma_{i}^{2}\left(\frac{k-1}{n}, X_{\frac{k-1}{n}}\right)\left(X_{\frac{k}{n}}-X_{\frac{k-1}{n}}\right)^{2}, \quad 1 \leq i \leq d .
\end{aligned}
$$


Recall that the quantity $\tilde{M}_{n}^{2}$ is based on a moderate frequency $1 / n$ (rather than $1 / N$ ), because return-based statistics computed at ultra-high frequencies are not adequate estimators in practice (due to microstructure noise). Dette, Podolskij \& Vetter (2006) have shown that $\sqrt{n}\left(\tilde{M}_{n}^{2}-M^{2}\right)$ is asymptotically mixed normal with conditional variance

$$
\tilde{\rho}^{2}=\left(1,-2\left(C_{1}, \ldots, C_{d}\right) D^{-1}\right) \int_{0}^{1} \tilde{\Sigma}_{s} d s\left(1,-2\left(C_{1}, \ldots, C_{d}\right) D^{-1}\right)^{T},
$$

where $\tilde{\Sigma}_{s}$ is defined by

$$
\tilde{\Sigma}_{s}=\left(\begin{array}{cccc}
\tilde{v}(s) & \tilde{v}_{1}(s) & \cdots & \tilde{v}_{d}(s) \\
\tilde{v}_{1}(s) & \tilde{v}_{11}(s) & \cdots & \tilde{v}_{1 d}(s) \\
\vdots & \vdots & \ddots & \vdots \\
\tilde{v}_{d}(s) & \tilde{v}_{d 1}(s) & \cdots & \tilde{v}_{d d}(s)
\end{array}\right)
$$

with

$$
\begin{aligned}
& \tilde{v}(s)=\frac{32}{3} \sigma^{8}\left(s, X_{s}\right), \\
& \tilde{v}_{i}(s)=4 \sigma_{i}^{2}\left(s, X_{s}\right) \sigma^{6}\left(s, X_{s}\right), \quad 1 \leq i \leq d \\
& \tilde{v}_{i j}(s)=2 \sigma_{i}^{2}\left(s, X_{s}\right) \sigma_{j}^{2}\left(s, X_{s}\right) \sigma^{4}\left(s, X_{s}\right), \quad 1 \leq i, j \leq d .
\end{aligned}
$$

By defining a return-based estimator $\tilde{\rho}_{n}^{2}$ of $\tilde{\rho}^{2}$ Dette, Podolskij \& Vetter (2006) have obtained a standard central limit theorem

$$
\frac{\sqrt{n}\left(\tilde{M}_{n}^{2}-M^{2}\right)}{\tilde{\rho}_{n}} \stackrel{\mathcal{D}}{\longrightarrow} N(0,1),
$$

which is used to construct the test for null hypothesis. Furthermore, Dette, Podolskij \& Vetter (2006) have also proposed to use a parametric bootstrap, similar to the described above, to improve the finite sample properties.

\subsection{Monte-Carlo simulations}

We investigate the performance of the bootstrap procedure for the problem of testing various linear hypotheses.

First, we demonstrate the simulated level of the range-based test obtained by applying the parametric bootstrap. In Tables 1-3 we present the results for the null hypothesis

$$
H_{0}: \sigma^{2}\left(t, X_{t}\right)=\alpha X_{t}^{2}
$$

and various drift functions $a(t, x)$. We generate the process $X$ from the stochastic differential equation (1) with $\sigma^{2}(t, x)=x^{2}$ and initial value $X_{0}=1$ (throughout this section we always choose $X_{0}=1$ ). The sample size (or the number of ranges) is $n=100,200,500$, while the number of observations used to compute the range is $m=10,20,50$. We use 1000 simulation 
runs and $B=500$ replications to simulate the rejection probabilities. We observe a rather accurate approximation for all drift functions even for small sample sizes. Quite surprisingly, the results do not depend much on $m$. In Table 7 we present the level approximation for the return-based test proposed by Dette, Podolskij \& Vetter (2006) (which corresponds to the case $m=1$ ). We observe similar approximation accuracy as demonstrated in Tables 1-3. Next, we present the corresponding results for the null hypothesis

$$
H_{0}: \sigma^{2}\left(t, X_{t}\right)=\alpha_{1}+\alpha_{2} X_{t}^{2}
$$

where the process $X$ is generated from the stochastic differential equation (1) with $\sigma^{2}(t, x)=$ $1+x^{2}$. In Tables 4-6 we state the simulated level of the range-based test while in Table 8 we present the simulated level of the return-based test. Again we conclude that the approximation accuracy does not significantly depend on $m$.

Next, we demonstrate the simulated power of the bootstrap method. In Tables 9-11 we present the simulated rejection probabilities for the null hypothesis of constant volatility and various alternatives. The drift function is $a(t, x)=(2-x) / 10$ and the simulation design is chosen as above. We observe a very high power of the range-based test even for $n=100$. Furthermore, the power is increasing in $m$. For the sake of comparison we present in Table 15 the corresponding results for the return-based test. The power of the return-based test is high; however, it is lower than the power of the range-based test at all sampling frequencies. This fact is not surprising since the return-based test corresponds to the case $m=1$.

The results look different for the simulated power for the null hypothesis

$$
H_{0}: \sigma^{2}\left(t, X_{t}\right)=\alpha X_{t}^{2}
$$

In Tables 12-14 we observe that the power of the range-based test crucially depends on the alternative. While we obtain rather good results for the alternative $\sigma^{2}(t, x)=1$, the power of the test for the alternative $\sigma^{2}(t, x)=5|x|^{3 / 2}$ is rather low and similar conclusions are true for the return-based test presented in Table 16. However, the low power for the alternative $\sigma^{2}(t, x)=5|x|^{3 / 2}$ is not very surprising. Clearly, for rather small values of the process $X$ (recall that $\left.X_{0}=1\right)$ the test can hardly detect the difference between the volatility functions of the form $|x|^{3 / 2}$ and $x^{2}$. Moreover, it is worthwhile to mention that the power increases in $m$, which implies that the range-based test is more powerful than the return-based test.

Finally, we demonstrate the simulated level and power of the test (22). First, note that we are not able to apply a bootstrap procedure, because we can not generate the data under $H_{0}: \omega \in \Omega_{0}(\epsilon)$. So we use the central limit theorem presented in Remark 3 as an approximation of the distribution of $\frac{M^{2}}{\left[\sigma^{2}, \sigma^{2}\right]}$. In Tables 17 and 18 we present the results for the null hypothesis

$$
H_{0}: \frac{M^{2}}{\left[\sigma^{2}, \sigma^{2}\right]} \leq 0.1
$$


with $\sigma^{2}(t, x)=(1+x)^{2}, a(t, x)=0$ and $\sigma_{1}^{2}(t, x)=x^{2}$. We use 5000 simulation runs to simulate the rejection probabilities. In each run we have to calculate in a first step if the null hypothesis holds (level simulation) or not (power simulation). Our choice of $\epsilon=0.1$ ensures that there are approximately the same number of level and power simulations. The number of ranges and their size are chosen as above. As expected from the inequality (23) we observe a quite conservative behaviour of the test (22) for all sample sizes. Moreover, the simulated level is decreasing in $m$ and $n$. On the other hand we obtain a reasonable power, which is increasing in $m$ and $n$. Note, however, that the corresponding bootstrap results for the hypothesis $H_{0}: M^{2}=0$ (Tables 12-14) are slightly better than those presented in Table 18, which can be explained by a better performance of the bootstrap method compared to a normal approximation.

\section{Appendix}

Proof of Theorem 3: First, let us note that we can assume (by stopping techniques and assumption (A1)) without loss of generality that the processes $a_{t}=a\left(t, X_{t}\right)$ and $\sigma_{t}=\sigma\left(t, X_{t}\right), \sigma_{1 t}=$ $\sigma_{1}\left(t, X_{t}\right), \ldots, \sigma_{d t}=\sigma_{d}\left(t, X_{t}\right)$ are bounded (see Barndorff-Nielsen, Graversen, Jacod, Podolskij \& Shephard (2006) for the justification of this assumption). Moreover, the constants that appear in the proofs are all denoted by $C$.

In Dette, Podolskij \& Vetter (2006) we have already shown that

$$
D^{n}-D=o_{p}\left(n^{-\frac{1}{2}}\right)
$$

and so we are left to proving the stable central limit theorem of Theorem 3. For this purpose we approximate the quantity $s_{i, n, m}(X)$ by $\left|\sigma_{\frac{i-1}{n}}\right| s_{i, n, m}(W)$. Indeed, Christensen \& Podolskij (2006b) have proven that under assumption (A2) the assertion

$$
\sqrt{n}\left(\begin{array}{c}
C_{0}^{n, m}-C_{0} \\
C_{1}^{n, m}-C_{1} \\
\vdots \\
C_{d}^{n, m}-C_{d}
\end{array}\right)=\left(\begin{array}{c}
\sum_{i=1}^{n}\left(\xi_{i, n, m}^{(0)}-E\left[\xi_{i, n, m}^{(0)} \mid \mathcal{F}_{\frac{i-1}{n}}\right]\right) \\
\sum_{i=1}^{n}\left(\xi_{i, n, m}^{(1)}-E\left[\xi_{i, n, m}^{(1)} \mid \mathcal{F}_{\frac{i-1}{n}}\right]\right) \\
\vdots \\
\sum_{i=1}^{n}\left(\xi_{i, n, m}^{(d)}-E\left[\xi_{i, n, m}^{(d)} \mid \mathcal{F}_{\frac{i-1}{n}}\right]\right)
\end{array}\right)+o_{p}\left(n^{-1 / 2}\right)
$$

with

$$
\begin{aligned}
\xi_{i, n, m}^{(0)} & =\frac{n^{3 / 2}}{\lambda_{4, m}} \sigma_{\frac{i-1}{n}}^{4} s_{i, n, m}^{4}(W) \\
\xi_{i, n, m}^{(k)} & =\frac{n^{1 / 2}}{\lambda_{2, m}} \sigma_{k, \frac{i-1}{n}}^{2} \sigma_{\frac{i-1}{n}}^{2} s_{i, n, m}^{2}(W) \quad k=1, \ldots, d,
\end{aligned}
$$

holds. Observe that

$$
\sum_{i=1}^{n}\left(E\left[\xi_{i, n, m}^{(k)} \xi_{i, n, m}^{(l)} \mid \mathcal{F}_{\frac{i-1}{n}}\right]-E\left[\xi_{i, n, m}^{(k)} \mid \mathcal{F}_{\frac{i-1}{n}}\right] E\left[\xi_{i, n, m}^{(l)} \mid \mathcal{F}_{\frac{i-1}{n}}\right]\right) \stackrel{P}{\longrightarrow} \int_{0}^{1} \Sigma_{s}^{k+1, l+1} d s
$$


for $0 \leq k, l \leq d$, and

$$
E\left[\left|\xi_{i, n, m}^{(k)}\right|^{4} \mid \mathcal{F}_{\frac{i-1}{n}}\right] \leq \frac{C}{n^{2}} \quad k=0, \ldots, d
$$

since the processes $\sigma_{t}, \sigma_{1 t}, \ldots, \sigma_{d t}$ are bounded. Next, notice that the identity

$$
E\left[\xi_{i, n, m}^{(k)}\left(W_{\frac{i}{n}}-W_{\frac{i-1}{n}}\right) \mid \mathcal{F}_{\frac{i-1}{n}}\right]=0 \quad k=0, \ldots, d
$$

holds, because the functionals $s_{i, n, m}^{2}(W)\left(W_{\frac{i}{n}}-W_{\frac{i-1}{n}}\right)$ and $s_{i, n, m}^{4}(W)\left(W_{\frac{i}{n}}-W_{\frac{i-1}{n}}\right)$ are both odd in $W$.

Finally, let $N$ be any bounded martingale on $\left(\Omega, \mathcal{F},\left(\mathcal{F}_{t}\right)_{t \in[0,1]}, P\right)$ which is orthogonal to $W$ (i.e. the covariation $\langle N, W\rangle_{t}=0$ a.s.). Then we deduce

$$
E\left[\xi_{i, n, m}^{(k)}\left(N_{\frac{i}{n}}-N_{\frac{i-1}{n}}\right) \mid \mathcal{F}_{\frac{i-1}{n}}\right]=0 \quad k=0, \ldots, d,
$$

which has been already shown in Christensen \& Podolskij (2006a). Now, the assertion of Theorem 3 follows from (25)-(29) and Theorem IX.7.28 in Jacod \& Shiryaev (2003).

Acknowledgements. The work of both authors was supported by the Deutsche Forschungsgemeinschaft (SFB 475, Komplexitätsreduktion in multivariaten Datenstrukturen).

\section{References}

[1] Achieser, N.I. (1956). Theory of approximation. New York, Frederick Ungar Publishing.

[2] Aldous, D.J., Eagleson, G.K. (1978). On mixing and stability of limit theorems. Ann. Probab. 6(2), 325-331.

[3] Barndorff-Nielsen, O.E., Graversen, S.E., Jacod, J., Podolskij, M., Shephard, N. (2006). A central limit theorem for realised power and bipower variations of continuous semimartingales. In Y. Kabanov, R. Lipster and J. Stoyanov, eds, "From Stochastic Analysis to Mathematical Finance, Festschrift for Albert Shiryaev", Springer, 33-68.

[4] Barndorff-Nielsen, O.E., Shephard, N. (2007). Variation, jumps, market frictions and high frequency data in financial econometrics. In R. Blundel, P. Torsten and W.K. Newey, eds, "Advances in economics and econometrics: theory and applications, ninth world congress" Cambridge University Press.

[5] Black, F., Scholes, M. (1973). The pricing of options and corporate liabilities. J. Polit. Econom. 81, 637-654.

[6] Chan, K.C., Karolyi, A.G., Longstaff, F.A., Sanders, A.B. (1992). An empirical comparison of alternative models of the short-term interest rate. Jour. Financ. 47, 1209-1227. 
[7] Christensen, K., Podolskij, M. (2006a). Realised range-based estimation of integrated variance. Forthcoming in J. Econometrics.

[8] Christensen, K., Podolskij, M. (2006b). Range-based estimation of quadratic variation. Working paper.

[9] Constantinides, G.M. (1992). A theory of the nominal term structure of interest rates. Rev. Finan. Studies 5, 531-552.

[10] Corradi, V., White, H. (1999). Specification tests for the variance of a diffusion. J. Time Ser. Anal. 20(3), 253-270.

[11] Cox, J.C., Ingersoll, J.E., Ross, S.A. (1985). A theory of the term structure of interest rate. Econometrica 53, 385-407.

[12] Dette, H., Podolskij, M. (2005). Testing the parametric form of the volatility in continuous time diffusion models - an empirical process approach. To apper in J. Econometrics

[13] Dette, H., Podolskij, M., Vetter, M. (2006). Estimation of integrated volatility in continuous time financial models with applications to goodness-of-fit testing. Scand. J. Statist. $33,259-278$.

[14] Dette, H., von Lieres und Wilkau, C. (2003). On a test for a parametric form of volatility in continuous time financial models. Finance Stoch. 7, 363-384.

[15] Feller, W. (1951). The asymptotic distribution of the range of sums of independent random variables. Ann. of Math. Stat. 22(3), 427-432.

[16] Hansen, P.R., Lunde, A. (2006). Realised variance and market microstructure noise. J. Bus. Econom. Statist. 24(2), 127-161.

[17] Hodges, J.L., Lehmann, E.L. (1954). Testing the approximative validity of statistical hypotheses. J. R. Stat. Soc. Ser. B Stat. Methodol. 16, 261-268.

[18] Jacod, J., Shiryaev, A.N. (2003). Limit theorems for stochastic processes. Berlin, Springer.

[19] Karatzas, I. (1988). On pricing of American options. Appl. Math. Optim. 17, 37-60.

[20] Karatzas, I., Shreve, S.E. (1991). Brownian motion and stochastic calculus. New York, Springer.

[21] Martens, M., van Dijk, D. (2005). Measuring volatility with the realised range. Working paper.

[22] Podolskij, M. (2006). New theory on estimation of integrated volatility with applications. PhD thesis. Ruhr-University Bochum, Department of Mathematics. 
[23] Renyi, A. (1963). On stable sequences of events. Sankya Ser. A 25, 293-302.

[24] Vasicek, O. A. (1977). An equilibrium characterization of the term structure. Jour. Financ. Econom. 5, 177-188. 
Table 1

\begin{tabular}{|c|c|c|c|c|c|c|c|c|c|}
\hline$\sigma^{2}(t, x)=x^{2}$ & \multicolumn{3}{|l|}{$n=100, m=10$} & \multicolumn{3}{|c|}{$n=200, m=10$} & \multicolumn{3}{|c|}{$n=500, m=10$} \\
\hline$a(t, x)$ & $20 \%$ & $10 \%$ & $5 \%$ & $20 \%$ & $10 \%$ & $5 \%$ & $20 \%$ & $10 \%$ & $5 \%$ \\
\hline 0 & 20.1 & 12.0 & 7.0 & 21.5 & 10.9 & 4.5 & 20.8 & 9.8 & 4.8 \\
0.2 & 20.7 & 11.3 & 5.2 & 21.9 & 11.8 & 6.9 & 21.9 & 11.3 & 6.4 \\
$x / 10$ & 21.6 & 10.9 & 5.4 & 20.2 & 10.1 & 5.6 & 20.3 & 10.0 & 5.3 \\
$(2-x) / 10$ & 20.6 & 9.6 & 5.0 & 19.1 & 9.9 & 4.1 & 22.7 & 12.6 & 6.4 \\
$t x$ & 25.8 & 13.8 & 7.4 & 23.7 & 12.5 & 7.1 & 23.5 & 12.3 & 7.1 \\
\hline
\end{tabular}

Table 2

\begin{tabular}{|c|c|c|c|c|c|c|c|c|c|}
\hline$\sigma^{2}(t, x)=x^{2}$ & \multicolumn{3}{|c|}{$n=100, m=20$} & \multicolumn{3}{|c|}{$n=200, m=20$} & \multicolumn{3}{|c|}{$n=500, m=20$} \\
\hline$a(t, x)$ & $20 \%$ & $10 \%$ & $5 \%$ & $20 \%$ & $10 \%$ & $5 \%$ & $20 \%$ & $10 \%$ & $5 \%$ \\
\hline 0 & 17.4 & 10.1 & 5.3 & 19.0 & 9.7 & 5.5 & 19.7 & 10.1 & 5.5 \\
0.2 & 21.6 & 11.5 & 6.5 & 22.8 & 12.1 & 6.1 & 20.4 & 11.2 & 6.6 \\
$x / 10$ & 17.9 & 9.7 & 5.1 & 22.9 & 11.5 & 5.3 & 22.7 & 11.6 & 6.4 \\
$(2-x) / 10$ & 20.7 & 10.9 & 5.1 & 22.8 & 12.6 & 6.8 & 21.2 & 11.4 & 6.6 \\
$t x$ & 26.4 & 14.1 & 7.7 & 25.7 & 12.5 & 6.4 & 20.5 & 11.1 & 5.8 \\
\hline
\end{tabular}

Table 3

\begin{tabular}{|c|c|c|c|c|c|c|c|c|c|}
\hline$\sigma^{2}(t, x)=x^{2}$ & \multicolumn{3}{|c|}{$n=100, m=50$} & \multicolumn{3}{|c|}{$n=200, m=50$} & \multicolumn{3}{|c|}{$n=500, m=50$} \\
\hline$a(t, x)$ & $20 \%$ & $10 \%$ & $5 \%$ & $20 \%$ & $10 \%$ & $5 \%$ & $20 \%$ & $10 \%$ & $5 \%$ \\
\hline 0 & 17.9 & 9.6 & 5.4 & 21.0 & 10.2 & 5.3 & 20.5 & 10.4 & 4.8 \\
0.2 & 19.8 & 10.1 & 5.1 & 21.7 & 11.6 & 5.8 & 20.0 & 10.1 & 5.3 \\
$x / 10$ & 24.5 & 11.5 & 6.2 & 23.9 & 12.4 & 7.3 & 19.9 & 11.4 & 6.3 \\
$(2-x) / 10$ & 20.5 & 10.3 & 4.8 & 21.2 & 10.2 & 5.5 & 20.8 & 10.1 & 3.9 \\
$t x$ & 24.5 & 14.2 & 7.4 & 25.1 & 13.0 & 7.3 & 22.0 & 11.2 & 5.4 \\
\hline
\end{tabular}

Table 1-3: Simulated level of the parametric bootstrap test based on the statistic $\sqrt{n} M_{N}^{2} / \rho_{N}$ for the hypothesis $H_{0}: \sigma^{2}\left(t, X_{t}\right)=\alpha X_{t}^{2}$ with $n=100,200,500, m=10,20,50$ and various drift functions. 
Table 4

\begin{tabular}{|c|c|c|c|c|c|c|c|c|c|}
\hline$\sigma^{2}(t, x)=1+x^{2}$ & \multicolumn{3}{|c|}{$n=100, m=10$} & \multicolumn{3}{|c|}{$n=200, m=10$} & \multicolumn{3}{|c|}{$n=500, m=10$} \\
\hline$a(t, x)$ & $20 \%$ & $10 \%$ & $5 \%$ & $20 \%$ & $10 \%$ & $5 \%$ & $20 \%$ & $10 \%$ & $5 \%$ \\
\hline 0 & 18.6 & 9.5 & 4.1 & 20.8 & 9.5 & 5.4 & 21.1 & 10.1 & 5.9 \\
0.2 & 22.4 & 12.0 & 6.3 & 19.7 & 10.0 & 5.6 & 24.1 & 11.2 & 6.5 \\
$x / 10$ & 21.0 & 10.5 & 5.0 & 19.8 & 10.2 & 5.4 & 19.0 & 9.8 & 5.0 \\
$(2-x) / 10$ & 20.4 & 10.4 & 5.6 & 20.4 & 10.3 & 5.2 & 20.3 & 10.2 & 4.7 \\
$t x$ & 21.0 & 10.2 & 5.4 & 23.5 & 12.3 & 7.0 & 20.3 & 9.4 & 4.5 \\
\hline
\end{tabular}

Table 5

\begin{tabular}{|c|c|c|c|c|c|c|c|c|c|}
\hline$\sigma^{2}(t, x)=1+x^{2}$ & \multicolumn{3}{|c|}{$n=100, m=20$} & \multicolumn{3}{|c|}{$n=200, m=20$} & \multicolumn{3}{|c|}{$n=500, m=20$} \\
\hline$a(t, x)$ & $20 \%$ & $10 \%$ & $5 \%$ & $20 \%$ & $10 \%$ & $5 \%$ & $20 \%$ & $10 \%$ & $5 \%$ \\
\hline 0 & 20.7 & 10.2 & 5.0 & 19.8 & 9.6 & 5.0 & 21.0 & 10.7 & 5.4 \\
0.2 & 20.5 & 10.2 & 5.0 & 21.2 & 11.1 & 6.1 & 21.1 & 11.2 & 6.3 \\
$x / 10$ & 20.9 & 10.2 & 5.2 & 20.7 & 10.6 & 5.4 & 19.4 & 10.2 & 4.3 \\
$(2-x) / 10$ & 17.7 & 7.6 & 3.5 & 17.6 & 8.1 & 4.9 & 20.1 & 10.0 & 6.2 \\
$t x$ & 23.0 & 11.4 & 6.6 & 21.6 & 11.6 & 5.9 & 25.7 & 12.8 & 6.2 \\
\hline
\end{tabular}

Table 6

\begin{tabular}{|c|c|c|c|c|c|c|c|c|c|}
\hline$\sigma^{2}(t, x)=1+x^{2}$ & \multicolumn{3}{|c|}{$n=100, m=50$} & \multicolumn{3}{|c|}{$n=200, m=50$} & \multicolumn{3}{|c|}{$n=500, m=50$} \\
\hline$a(t, x)$ & $20 \%$ & $10 \%$ & $5 \%$ & $20 \%$ & $10 \%$ & $5 \%$ & $20 \%$ & $10 \%$ & $5 \%$ \\
\hline 0 & 20.2 & 9.9 & 5.6 & 18.9 & 9.5 & 5.3 & 22.2 & 11.6 & 6.1 \\
0.2 & 22.4 & 10.8 & 6.0 & 20.1 & 11.1 & 5.4 & 21.5 & 10.5 & 5.2 \\
$x / 10$ & 18.8 & 8.1 & 4.1 & 21.1 & 11.4 & 5.8 & 20.9 & 10.6 & 6.9 \\
$(2-x) / 10$ & 19.5 & 9.3 & 5.5 & 18.9 & 8.6 & 3.8 & 19.1 & 9.8 & 4.7 \\
$t x$ & 25.7 & 13.9 & 6.5 & 23.9 & 11.6 & 6.6 & 21.4 & 11.7 & 5.1 \\
\hline
\end{tabular}

Table 4-6: Simulated level of the parametric bootstrap test based on the statistic $\sqrt{n} M_{N}^{2} / \rho_{N}$ for the hypothesis $H_{0}: \sigma^{2}\left(t, X_{t}\right)=\alpha_{1}+\alpha_{2} X_{t}^{2}$ with $n=100,200,500, m=10,20,50$ and various drift functions. 
Table 7

\begin{tabular}{|c|c|c|c|c|c|c|c|c|c|}
\hline$\sigma^{2}(t, x)=x^{2}$ & \multicolumn{3}{|c|}{$n=100$} & \multicolumn{3}{c|}{$n=200$} & \multicolumn{3}{c|}{$n=500$} \\
\hline$a(t, x)$ & $20 \%$ & $10 \%$ & $5 \%$ & $20 \%$ & $10 \%$ & $5 \%$ & $20 \%$ & $10 \%$ & $5 \%$ \\
\hline 0 & 19.8 & 11.8 & 6.9 & 21.0 & 12.1 & 5.8 & 20.2 & 10.5 & 5.8 \\
0.2 & 22.4 & 11.3 & 5.5 & 20.2 & 8.8 & 5.0 & 23.0 & 11.4 & 6.2 \\
$x / 10$ & 22.6 & 11.4 & 5.9 & 21.3 & 9.8 & 4.8 & 20.1 & 10.4 & 5.5 \\
$(2-x) / 10$ & 20.9 & 10.2 & 5.1 & 18.8 & 9.8 & 4.3 & 22.4 & 12.5 & 6.2 \\
$t x$ & 26.6 & 14.7 & 8.4 & 23.8 & 13.4 & 8.3 & 23.1 & 12.4 & 6.3 \\
\hline
\end{tabular}

Table 7: Simulated level of the parametric bootstrap test based on the statistic $\sqrt{n} \tilde{M}_{n}^{2} / \tilde{\rho}_{n}$ for the hypothesis $H_{0}: \sigma^{2}\left(t, X_{t}\right)=\alpha X_{t}^{2}$ with $n=100,200,500$ and various drift functions.

Table 8

\begin{tabular}{|c|c|c|c|c|c|c|c|c|c|}
\hline$\sigma^{2}(t, x)=1+x^{2}$ & \multicolumn{3}{|c|}{$n=100$} & \multicolumn{3}{c|}{$n=200$} & \multicolumn{3}{c|}{$n=500$} \\
\hline$a(t, x)$ & $20 \%$ & $10 \%$ & $5 \%$ & $20 \%$ & $10 \%$ & $5 \%$ & $20 \%$ & $10 \%$ & $5 \%$ \\
\hline 0 & 19.4 & 8.6 & 4.3 & 20.3 & 9.1 & 4.6 & 20.4 & 10.2 & 5.3 \\
0.2 & 22.4 & 12.9 & 6.9 & 19.3 & 11.0 & 6.7 & 23.5 & 11.2 & 5.8 \\
$x / 10$ & 21.7 & 11.1 & 5.3 & 21.3 & 10.6 & 4.9 & 21.4 & 10.2 & 5.3 \\
$(2-x) / 10$ & 22.4 & 10.9 & 5.8 & 20.1 & 9.9 & 4.9 & 18.4 & 9.3 & 4.8 \\
$t x$ & 21.9 & 12.3 & 6.8 & 23.2 & 13.3 & 7.1 & 22.2 & 10.7 & 5.6 \\
\hline
\end{tabular}

Table 8: Simulated level of the parametric bootstrap test based on the statistic $\sqrt{n} \tilde{M}_{n}^{2} / \tilde{\rho}_{n}$ for the hypothesis $H_{0}: \sigma^{2}\left(t, X_{t}\right)=\alpha_{1}+\alpha_{2} X_{t}^{2}$ with $n=100,200,500$ and various drift functions. 
Table 9

\begin{tabular}{|c|c|c|c|c|c|c|c|c|c|}
\hline$a(t, x)=(2-x) / 10$ & \multicolumn{3}{|c|}{$n=100, m=10$} & \multicolumn{3}{c|}{$n=200, m=10$} & \multicolumn{3}{c|}{$n=500, m=10$} \\
\hline$\sigma^{2}(t, x)$ & $20 \%$ & $10 \%$ & $5 \%$ & $20 \%$ & $10 \%$ & $5 \%$ & $20 \%$ & $10 \%$ & $5 \%$ \\
\hline $1+x^{2}$ & 93.0 & 88.4 & 84.7 & 96.9 & 95.1 & 93.0 & 98.9 & 98.0 & 97.2 \\
$x^{2}$ & 99.4 & 98.3 & 97.2 & 99.9 & 99.9 & 99.8 & 100.0 & 100.0 & 100.0 \\
$5|x|^{3 / 2}$ & 100.0 & 100.0 & 100.0 & 100.0 & 100.0 & 100.0 & 100.0 & 100.0 & 100.0 \\
$5|x|$ & 100.0 & 100.0 & 100.0 & 100.0 & 100.0 & 100.0 & 100.0 & 100.0 & 100.0 \\
$(1+x)^{2}$ & 100.0 & 100.0 & 100.0 & 100.0 & 100.0 & 100.0 & 100.0 & 100.0 & 100.0 \\
\hline
\end{tabular}

Table 10

\begin{tabular}{|c|c|c|c|c|c|c|c|c|c|}
\hline$a(t, x)=(2-x) / 10$ & \multicolumn{3}{|c|}{$n=100, m=20$} & \multicolumn{3}{c|}{$n=200, m=20$} & \multicolumn{3}{c|}{$n=500, m=20$} \\
\hline$\sigma^{2}(t, x)$ & $20 \%$ & $10 \%$ & $5 \%$ & $20 \%$ & $10 \%$ & $5 \%$ & $20 \%$ & $10 \%$ & $5 \%$ \\
\hline $1+x^{2}$ & 94.2 & 89.7 & 86.2 & 96.7 & 95.2 & 92.9 & 99.6 & 99.4 & 98.2 \\
$x^{2}$ & 99.8 & 99.5 & 98.6 & 99.8 & 99.7 & 99.7 & 100.0 & 100.0 & 100.0 \\
$5|x|^{3 / 2}$ & 100.0 & 100.0 & 100.0 & 100.0 & 100.0 & 100.0 & 100.0 & 100.0 & 100.0 \\
$5|x|$ & 100.0 & 100.0 & 100.0 & 100.0 & 100.0 & 100.0 & 100.0 & 100.0 & 100.0 \\
$(1+x)^{2}$ & 100.0 & 100.0 & 100.0 & 100.0 & 100.0 & 100.0 & 100.0 & 100.0 & 100.0 \\
\hline
\end{tabular}

Table 11

\begin{tabular}{|c|c|c|c|c|c|c|c|c|c|}
\hline$a(t, x)=(2-x) / 10$ & \multicolumn{3}{|c|}{$n=100, m=50$} & \multicolumn{3}{c|}{$n=200, m=50$} & \multicolumn{3}{c|}{$n=500, m=50$} \\
\hline$\sigma^{2}(t, x)$ & $20 \%$ & $10 \%$ & $5 \%$ & $20 \%$ & $10 \%$ & $5 \%$ & $20 \%$ & $10 \%$ & $5 \%$ \\
\hline $1+x^{2}$ & 94.1 & 90.8 & 88.3 & 97.5 & 95.8 & 93.9 & 99.5 & 98.8 & 97.8 \\
$x^{2}$ & 99.6 & 99.1 & 98.8 & 100.0 & 100.0 & 100.0 & 100.0 & 100.0 & 100.0 \\
$5|x|^{3 / 2}$ & 100.0 & 100.0 & 100.0 & 100.0 & 100.0 & 100.0 & 100.0 & 100.0 & 100.0 \\
$5|x|$ & 100.0 & 100.0 & 100.0 & 100.0 & 100.0 & 100.0 & 100.0 & 100.0 & 100.0 \\
$(1+x)^{2}$ & 100.0 & 100.0 & 100.0 & 100.0 & 100.0 & 100.0 & 100.0 & 100.0 & 100.0 \\
\hline
\end{tabular}

Table 9-11: Simulated power of the parametric bootstrap test based on the statistic $\sqrt{n} M_{N}^{2} / \rho_{N}$ for the hypothesis $H_{0}: \sigma^{2}\left(t, X_{t}\right)=\alpha$ with $n=100,200,500, m=10,20,50$ and various alternatives. 
Table 12

\begin{tabular}{|c|c|c|c|c|c|c|c|c|c|}
\hline$a(t, x)=(2-x) / 10$ & \multicolumn{3}{|c|}{$n=100, m=10$} & \multicolumn{3}{c|}{$n=200, m=10$} & \multicolumn{3}{|c|}{$n=500, m=10$} \\
\hline$\sigma^{2}(t, x)$ & $20 \%$ & $10 \%$ & $5 \%$ & $20 \%$ & $10 \%$ & $5 \%$ & $20 \%$ & $10 \%$ & $5 \%$ \\
\hline $1+x^{2}$ & 69.8 & 55.3 & 40.5 & 68.2 & 54.4 & 46.3 & 72.7 & 61.1 & 55.6 \\
1 & 91.9 & 86.6 & 79.8 & 95.9 & 92.2 & 88.4 & 98.4 & 97.0 & 95.3 \\
$5|x|^{3 / 2}$ & 33.8 & 18.5 & 11.1 & 39.0 & 20.0 & 11.5 & 42.0 & 23.3 & 12.4 \\
$5|x|$ & 55.3 & 38.3 & 22.6 & 63.1 & 44.4 & 30.0 & 79.0 & 61.8 & 45.1 \\
$(1+x)^{2}$ & 68.4 & 50.1 & 33.4 & 75.9 & 60.6 & 48.2 & 79.2 & 68.3 & 57.1 \\
\hline
\end{tabular}

Table 13

\begin{tabular}{|c|c|c|c|c|c|c|c|c|c|}
\hline$a(t, x)=(2-x) / 10$ & \multicolumn{3}{|c|}{$n=100, m=20$} & \multicolumn{3}{c|}{$n=200, m=20$} & \multicolumn{3}{|c|}{$n=500, m=20$} \\
\hline$\sigma^{2}(t, x)$ & $20 \%$ & $10 \%$ & $5 \%$ & $20 \%$ & $10 \%$ & $5 \%$ & $20 \%$ & $10 \%$ & $5 \%$ \\
\hline $1+x^{2}$ & 69.6 & 53.6 & 40.3 & 68.8 & 57.6 & 48.6 & 73.5 & 65.2 & 57.3 \\
1 & 92.5 & 87.6 & 82.6 & 97.1 & 94.0 & 91.6 & 99.2 & 98.4 & 96.7 \\
$5|x|^{3 / 2}$ & 34.0 & 18.1 & 9.5 & 34.6 & 20.6 & 10.5 & 36.2 & 19.7 & 9.7 \\
$5|x|$ & 55.1 & 34.7 & 20.8 & 63.5 & 45.8 & 29.3 & 79.0 & 61.9 & 46.3 \\
$(1+x)^{2}$ & 70.4 & 50.4 & 34.3 & 74.5 & 60.3 & 44.6 & 80.3 & 67.9 & 56.0 \\
\hline
\end{tabular}

Table 14

\begin{tabular}{|c|c|c|c|c|c|c|c|c|c|}
\hline$a(t, x)=(2-x) / 10$ & \multicolumn{3}{|c|}{$n=100, m=50$} & \multicolumn{3}{|c|}{$n=200, m=50$} & \multicolumn{3}{|c|}{$n=500, m=50$} \\
\hline$\sigma^{2}(t, x)$ & $20 \%$ & $10 \%$ & $5 \%$ & $20 \%$ & $10 \%$ & $5 \%$ & $20 \%$ & $10 \%$ & $5 \%$ \\
\hline $1+x^{2}$ & 69.1 & 55.2 & 44.3 & 71.0 & 59.6 & 49.0 & 73.5 & 64.8 & 57.5 \\
1 & 95.3 & 90.0 & 85.0 & 97.7 & 94.9 & 92.8 & 99.5 & 98.6 & 97.9 \\
$5|x|^{3 / 2}$ & 31.6 & 16.6 & 7.6 & 33.3 & 16.0 & 8.1 & 35.9 & 20.7 & 11.5 \\
$5|x|$ & 49.3 & 32.2 & 17.6 & 63.2 & 46.9 & 30.7 & 77.0 & 62.7 & 47.8 \\
$(1+x)^{2}$ & 72.1 & 51.3 & 33.1 & 76.8 & 63.8 & 50.0 & 79.7 & 69.9 & 60.3 \\
\hline
\end{tabular}

Table 12-14: Simulated power of the parametric bootstrap test based on the statistic $\sqrt{n} M_{N}^{2} / \rho_{N}$ for the hypothesis $H_{0}: \sigma^{2}\left(t, X_{t}\right)=\alpha X_{t}^{2}$ with $n=100,200,500, m=10,20,50$ and various alternatives. 
Table 15

\begin{tabular}{|c|c|c|c|c|c|c|c|c|c|}
\hline$a(t, x)=(2-x) / 10$ & \multicolumn{3}{|c|}{$n=100$} & \multicolumn{3}{c|}{$n=200$} & \multicolumn{3}{c|}{$n=500$} \\
\hline$\sigma^{2}(t, x)$ & $20 \%$ & $10 \%$ & $5 \%$ & $20 \%$ & $10 \%$ & $5 \%$ & $20 \%$ & $10 \%$ & $5 \%$ \\
\hline $1+x^{2}$ & 80.5 & 73.3 & 67.9 & 88.0 & 84.0 & 80.2 & 94.1 & 92.4 & 90.3 \\
$x^{2}$ & 94.3 & 91.4 & 88.9 & 98.9 & 97.6 & 96.4 & 100.0 & 99.6 & 99.4 \\
$5|x|^{3 / 2}$ & 100.0 & 100.0 & 100.0 & 100.0 & 100.0 & 100.0 & 100.0 & 100.0 & 100.0 \\
$5|x|$ & 100.0 & 100.0 & 100.0 & 100.0 & 100.0 & 100.0 & 100.0 & 100.0 & 100.0 \\
$(1+x)^{2}$ & 99.8 & 99.6 & 98.8 & 100.0 & 100.0 & 99.9 & 100.0 & 100.0 & 100.0 \\
\hline
\end{tabular}

Table 15: Simulated power of the parametric bootstrap test based on the statistic $\sqrt{n} \tilde{M}_{N}^{2} / \tilde{\rho}_{n}$ for the hypothesis $H_{0}: \sigma^{2}\left(t, X_{t}\right)=\alpha$ with $n=100,200,500$ and various alternatives.

Table 16

\begin{tabular}{|c|c|c|c|c|c|c|c|c|c|}
\hline$a(t, x)=(2-x) / 10$ & \multicolumn{3}{|c|}{$n=100$} & \multicolumn{3}{c|}{$n=200$} & \multicolumn{3}{c|}{$n=500$} \\
\hline$\sigma^{2}(t, x)$ & $20 \%$ & $10 \%$ & $5 \%$ & $20 \%$ & $10 \%$ & $5 \%$ & $20 \%$ & $10 \%$ & $5 \%$ \\
\hline $1+x^{2}$ & 58.8 & 44.5 & 35.6 & 58.3 & 46.4 & 39.0 & 65.8 & 56.8 & 51.5 \\
1 & 80.7 & 73.1 & 67.2 & 86.6 & 82.0 & 77.8 & 94.0 & 90.6 & 88.0 \\
$5|x|^{3 / 2}$ & 36.0 & 20.2 & 11.7 & 37.0 & 20.0 & 10.2 & 36.4 & 20.2 & 12.2 \\
$5|x|$ & 51.2 & 34.4 & 24.3 & 54.2 & 34.8 & 25.0 & 60.3 & 45.3 & 35.4 \\
$(1+x)^{2}$ & 57.0 & 39.7 & 28.9 & 64.0 & 47.8 & 37.7 & 66.0 & 55.5 & 48.6 \\
\hline
\end{tabular}

Table 16: Simulated power of the parametric bootstrap test based on the statistic $\sqrt{n} \tilde{M}_{n}^{2} / \tilde{\rho}_{n}$ for the hypothesis $H_{0}: \sigma^{2}\left(t, X_{t}\right)=\alpha X_{t}^{2}$ with $n=100,200,500$ and various alternatives. 
Table 17

\begin{tabular}{|c|c|c|c|c|c|c|c|c|c|}
\hline$a(t, x)=0$ & \multicolumn{3}{|c|}{$n=100$} & \multicolumn{3}{c|}{$n=200$} & \multicolumn{3}{c|}{$n=500$} \\
\hline$\sigma^{2}(t, x)=(1+x)^{2}$ & $20 \%$ & $10 \%$ & $5 \%$ & $20 \%$ & $10 \%$ & $5 \%$ & $20 \%$ & $10 \%$ & $5 \%$ \\
\hline $\mathrm{m}=10$ & 8.4 & 4.4 & 2.7 & 7.1 & 3.1 & 1.4 & 4.4 & 2.0 & 1.1 \\
$\mathrm{~m}=20$ & 8.0 & 3.8 & 1.5 & 6.0 & 2.4 & 1.1 & 4.9 & 1.6 & 0.6 \\
$\mathrm{~m}=50$ & 6.9 & 3.3 & 1.6 & 6.4 & 2.6 & 1.3 & 3.8 & 1.4 & 0.5 \\
\hline
\end{tabular}

Table 17: Simulated level of the test (22) for the hypothesis $H_{0}: \frac{M^{2}}{\left[\sigma^{2}, \sigma^{2}\right]} \leq 0.1$ with $\sigma_{1}^{2}(t, x)=x^{2}, n=100,200,500$ and $m=10,20,50$.

Table 18

\begin{tabular}{|c|c|c|c|c|c|c|c|c|c|}
\hline$a(t, x)=0$ & \multicolumn{3}{|c|}{$n=100$} & \multicolumn{3}{c|}{$n=200$} & \multicolumn{3}{c|}{$n=500$} \\
\hline$\sigma^{2}(t, x)=(1+x)^{2}$ & $20 \%$ & $10 \%$ & $5 \%$ & $20 \%$ & $10 \%$ & $5 \%$ & $20 \%$ & $10 \%$ & $5 \%$ \\
\hline $\mathrm{m}=10$ & 46.6 & 36.7 & 31.1 & 56.2 & 45.3 & 38.6 & 69.6 & 61.9 & 55.8 \\
$\mathrm{~m}=20$ & 48.7 & 39.2 & 32.9 & 60.9 & 51.2 & 44.3 & 69.7 & 61.3 & 55.0 \\
$\mathrm{~m}=50$ & 52.7 & 43.1 & 35.9 & 61.6 & 52.2 & 45.1 & 73.5 & 65.8 & 59.5 \\
\hline
\end{tabular}

Table 18: Simulated power of the test (22) for the hypothesis $H_{0}: \frac{M^{2}}{\left[\sigma^{2}, \sigma^{2}\right]} \leq 0.1$ with $\sigma_{1}^{2}(t, x)=x^{2}, n=100,200,500$ and $m=10,20,50$. 